\title{
Author Correction: Cross-species single-cell transcriptomic analysis reveals pre-gastrulation developmental differences among pigs, monkeys, and humans
}

\author{
Tianbin Liu, Jie Li, Leqian Yu, Hai-Xi Sun, Jing Li, Guoyi Dong, Yingying Hu, Yong Li, Yue Shen (D, Jun Wu (1D and \\ Ying Gu (D)
}

Correction to: Cell Discovery (2021) 7:8

https://doi.org/10.1038/s41421-020-00238-x

Published online 02 February 2021

In the original publication of this article ${ }^{1}$, the description of data source and reference for the identification of epithelial cell lineages of pigs were missing and should be added in Materials and methods section as follows.

\section{Lineage identification of cells in Pig}

The expression matrix of the endometrial epithelium, including the pregnant glandular epithelium (GSM294604144), the pregnant luminal epithelium (GSM2946045-48), the control glandular epithelium (GSM2946030-33), and the control luminal epithelium (GSM2946034-37), was downloaded from GSE109539².

The newly added reference ${ }^{2}$ should be cited in the main text as shown below.
"To investigate potential roles of the endometrial epithelium may play in pig embryo elongation, we used CellPhone $\mathrm{DB}^{45}$ to predict the ligand-receptor interactions between pregnant womb epithelium (luminal and glandular, P_LE and P_GE) ${ }^{2}$ and PostL-EPIs or PostL-TEs during the elongation stages".

In addition, in the last Venn diagram of Fig. 5c, the labels of 52 and 169 were misplaced and should be interchanged as shown below.

Published online: 12 March 2021

\section{References}

1. Liu, T. et al. Cross-species single-cell transcriptomic analysis reveals pregastrulation developmental differences among pigs, monkeys, and humans. Cell Discov. 7, 8 (2021).

2. Zeng, S., Bick, J., Ulbrich, S. E. \& Bauersachs, S. Cell type-specific analysis of transcriptome changes in the porcine endometrium on Day 12 of pregnancy. BMC Genomics 19, 459 (2018).

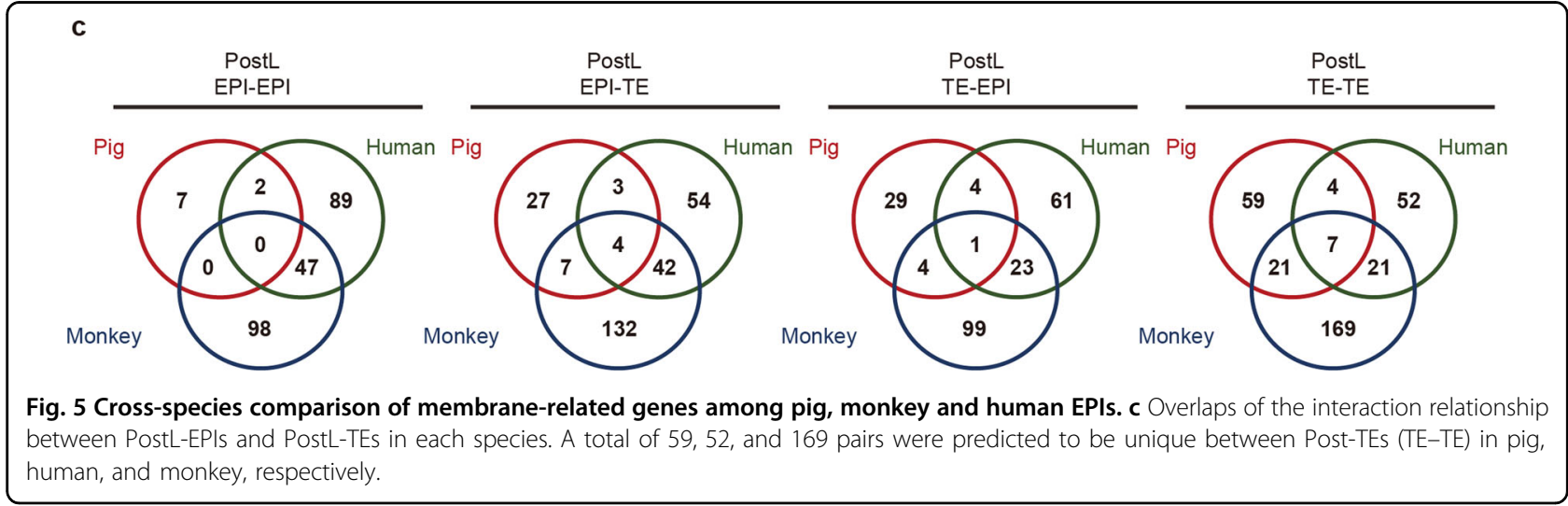

